Daimon. Revista Internacional de Filosofía, no 84, 2021 pp. 131-145

ISSN: 1130-0507 (papel) y 1989-4651 (electrónico)

http://dx.doi.org/10.6018/daimon.463831

Licencia Creative Commons Reconocimiento-NoComercial-SinObraDerivada 3.0 España (texto legal). Se pueden copiar, usar, difundir, transmitir y exponer públicamente, siempre que: i) se cite la autoría y la fuente original de su publicación (revista, editorial y URL de la obra); ii) no se usen para fines comerciales; iii) se mencione la existencia y especificaciones de esta licencia de uso.

(c) (1) (9)

\title{
Las cualidades secundarias y el valor epistémico de la experiencia estética
}

\section{Secondary Qualities and the Epistemic Value of Aesthetic Experience}

NIEVES ACEDO*

\begin{abstract}
Resumen: Entendido lo estético como vinculado al carácter apreciativo de la experiencia sensible, el presente artículo explora su rescate del ámbito de la mera opinión vinculándolo con la intencionalidad de la sensación. Tras definir el problema en la introducción, el texto se centra en las llamadas cualidades secundarias, objeto inmediato de la sensación. Se parte de la hipótesis de que el débil contenido epistémico que se ha atribuido a las cualidades secundarias a lo largo de la historia es responsable de la difícil valoración del juicio estético. Se procede por ello a una reformulación del tipo de noticia que recibimos de dichas cualidades, partiendo de la definición de cualidad secundaria de John Locke, y revisándola a partir de algunos textos de John McDowell y Crispin Wright, en diálogo con la teoría aristotélica de los sensibles propios. El resultado de esta revisión afectará al papel que atribuyamos, dentro del espectro de las disciplinas, a la estética y, secundariamente, al arte.

Palabras clave: teoría de la percepción, cualidades secundarias, estética empirista, epistemología de la sensación, John McDowell, Crispin Wright.
\end{abstract}

\begin{abstract}
Considering Aesthetics as linked to the appreciative nature of sensorial experience, this article uses the concept of intentionality of sensation to rescue Aesthetics from being confined into the scope of mere opinions. After introducing and defining the problem, the text focuses on the so- called secondary qualities, immediate object of the sensation. The hypothesis is that the weak epistemic content attributed to secondary qualities throughout history is responsible for the difficult assessment of aesthetic judgment. A reformulation of the kind of news we receive from these qualities is proposed reviewing John McDowell and Crispin Wright review of Lockean's secondary qualities, in dialogue with the Aristotelian theory of the proper sensible. The result of this review should influence the role we attribute, within the spectrum of disciplines, to Aesthetics and, secondarily, to Art.
\end{abstract}

Keywords: theory of perception, secondary qualities, empirical aesthetics, epistemology of sensation.

Recibido: 16/01/2021 Aceptado: 26/05/2021.

* Profesora colaboradora en el Departamento de Historia, Arte y Geografía de la Universidad de Navarra. nacedo@unav.es. Líneas de investigación: teoría del arte, estudios curatoriales, arte y sociedad. Publicaciones recientes: Acedo, Nieves (2018): "Los Tapies del Museo Universidad de Navarra: el estilo como frontera entre lo internacional y lo identitario", Príncipe de Viana, Año LXXIX, n. . 272, pp. 1307-1322; Acedo, Nieves (2019): "Arte contemporáneo y verdad. Estrategias para el desengaño", La posverdad o el dominio de lo trivial, Flamarique y Carbonell (eds.), Encuentro, Madrid, pp.180-194. 


\section{El problema de la circularidad arte-estética}

La estética ocupa un lugar inestable e incómodo en el complejo mapa de las disciplinas debido a la cambiante definición de su objeto. Como teoría de lo bello, según la definición clásica de la belleza como trascendental del ente, formó parte de la ciencia del ser, en cuanto que la belleza es convertible con este último. Lo específico de la estética se buscaba en la diferencia de la propiedad trascendental "bello" con respecto a las otras propiedades (bueno, verdadero...) que son igualmente convertibles con el ser, pero diversas entre sí. Se encontró así que una de las notas diferenciales de lo bello es su carácter relacional. En línea con este aspecto relacional, ya en una época marcada por el giro copernicano hacia el sujeto, la idea de lo bello se buscó investigando el tipo de emoción que despierta. Así, coincidiendo con la definición de la estética como disciplina independiente en el siglo XVIII entró en juego otra idea de lo bello que, siguiendo el cauce del pensamiento moderno, tiempo después acabó por excluir la belleza natural, abriendo paso a la idea de que lo bello tiene su lugar propio en el ámbito del arte. Por su parte, desde las vanguardias, la praxis del arte quiso desvincularse de la conexión con lo bello, de la que tal vez había abusado el academicismo. De este modo, lo artístico desligado de lo bello llegó a adquirir protagonismo en los estudios de estética, hasta el punto de que hoy ya no es fácil diferenciar la estética de la teoría del arte.

En contra de esta identificación, la teoría del arte se separa de la estética precisamente porque acude a ella para evitar la disolución del hecho artístico en las cambiantes configuraciones culturales a la que conduce la mera descripción historicista. Así, el arte busca en la estética una ontología que responda a la pregunta por el modo de ser específico de lo artístico, un modo de ser o "artisticidad" en el que a su vez radica aquello "estético", objeto de la estética.

Como se ve, la deriva moderna de la estética hacia la teoría del arte cae en un planteamiento circular: el arte queda indefinido porque no se puede clarificar a partir de una estética que busca en lo artístico su propia definición. En otros términos, el núcleo estético de lo artístico exige atribuir a la estética un objeto diverso del arte; "lo estético" no puede ser primariamente "lo artístico", sino alguna otra cosa. Para superar la aporía de esta circularidad será útil volver al punto de partida de la moderna disciplina estética, que ya no está centrada en la belleza como trascendental, pero tampoco se limita a lo productivo (poíesis), sino que está interesada sobre todo en la experiencia o la apreciación (aísthesis). Y pensar la experiencia estética nos coloca en el ámbito de la percepción sensible.

Fenomenológicamente, la sensación aparece como base de la experiencia estética en cuanto que reporta una noticia que no conduce a la conceptualización y que, sin ser irracional - sino en todo caso "pre-comprensiva" - percibimos como próxima a lo afectivo. No significa esto una reducción de la experiencia estética a lo inmediatamente sensible, porque a las cualidades, a lo particular de la experiencia del mundo, a las formalidades del aparecer, se añade la mediación de las configuraciones culturales que permiten reconocer en las apariencias otros plexos de relaciones distintos al inmediato manifestarse del objeto que también configuran la complacencia o el rechazo. Se podría definir así un logos de lo estético en el que cada experiencia particular, además de permitir el conocimiento según una razón científica, se formaliza para seguir mediando de modo acumulativo el placer y el displacer de nuestra relación con el mundo. El receptáculo que somos no es por tanto neutro, sino ya configurado y siempre configurándose de un modo que es histórico-contingente. 
Dicho de otro modo, la sensación no se agota en la operación de tipo inmanente que es el conocimiento, sino que es materia para procesos transitivos en los que se formaliza y se almacena como material que media las subsiguientes sensaciones.

Ahora bien, aunque transitiva y no inmanente, la percepción sensible no es pasiva, sino que es también operación, inmutación activa. Este carácter activo lo encontramos ya en Aristóteles, para quien la sensación es operación o acto segundo del alma $\left(D A, 418^{\mathrm{a}}\right)^{1}$ y en Tomás de Aquino, que abunda en ello al exigir, para que se dé la sensación, un tipo de alteración activa que llama "espiritual" ( $S T, \mathrm{I}, 78,3)$. La cuestión es importante porque, como se sabe, no hay objeto intencional sin acto. Por lo tanto, del reconocimiento del carácter activo de la sensación depende la intencionalidad de su objeto. El empirismo representacionista de John Locke desplaza la parte activa de la sensación a la reflexión que acompaña y refuerza la impresión $(E, \mathrm{II}, 9,15)$ de tal modo que el pensamiento posterior con frecuencia da por hecho su pasividad. Cabe sin embargo, como veremos, postular la intencionalidad del objeto de la percepción, lo que afecta no sólo al alcance del conocimiento sensible, sino también a la adecuada comprensión del aspecto estético.

Como dijimos, la experiencia estética viene también definida por el componente apreciativo que se advierte en la sensación. Así, por ejemplo, el aprecio por la específica configuración sensible del momento presente, que percibo con un agrado que ignora el simple bienestar: esa vista y su luz cambiante, el peculiar timbre, tono y volumen de esas voces, inseparables en mi experiencia de este frío y este olor particular, aquí, en esta sala. De modo que entendemos lo estético como el aspecto que dota a la experiencia sensible de un elemento apreciativo y judicativo, que tiene su base, como el conocimiento, en la inmutación activa que es la sensación. La cuestión es que mi juicio estético aprobatorio del momento presente, por seguir con el ejemplo anterior, aparece vinculado por una parte con lo particular sensible - resistente a la generalización - y por otro con la mencionada formalización de mi sensibilidad a partir de configuraciones culturales - históricamente relativas - , y ambos factores parecen relegar tal juicio al ámbito meramente subjetivo de las opiniones, donde no es posible encontrar ningún contenido que fundamente la carga de verdad que se pone en juego al juzgar.

Ahora bien, puesto que tanto en el juicio teórico como en el práctico comparece un elemento estético, y siendo así que la vida política, la convivencia social, el reconocimiento de las identidades, etc., están atravesados por cuestiones estéticas, importa llegar a definir el papel de la experiencia estética en el proceso de juzgar. Teniendo en cuenta para ello lo señalado sobre la intencionalidad de la sensación, nos disponemos a utilizar este elemento como palanca de rescate del juicio estético del ámbito de la opinión.

Para ello en lo que sigue nos centraremos en las llamadas cualidades secundarias, que la escuela empirista definió como el objeto inmediato de la sensación, procediendo en primer lugar a describir la taxonomía de las cualidades sensibles de John Locke. En segundo lugar, nos referiremos a la reciente revisión de esta taxonomía por parte de filósofos analíticos, en particular John McDowell y Crispin Wright.

1 En los autores de los que existe una edición canónica de la obra completa se ha optado por citar el texto en castellano (según las ediciones que aparecen en la bibliografía), acompañado de la numeración canónica correspondiente. 
Pero antes de proceder a este análisis conviene señalar que la defensa de la objetividad o intersubjetividad de la experiencia estética no es una preocupación nueva o aislada. De hecho, lo general en la reflexión filosófica ha sido no resignarse a la idea de que la determinación estética sea sólo un aspecto concomitante del juicio y, por tanto, potencialmente perturbador, obstáculo a su rectitud. Así, el empeño en rescatar el juicio estético del relativismo subjetivo fue una de las principales tareas a las que se dedicó la estética moderna. Especialmente destacados fueron los esfuerzos en el entorno empirista que intentaron demostrar la posibilidad de un "placer desinteresado" para justificar la imparcialidad del juicio de gusto (desinteresado en cuanto que la voluntad no busca poseerlo).

La teoría kantiana se desmarcó en parte del empirismo, pero asumió algunas de sus soluciones, como la del mencionado "placer desinteresado" que se despierta ante ciertos objetos o representaciones y que permite distinguir lo bello de lo bueno. Así en la Crítica del juicio (1790) se dice: "Gusto es la facultad de juzgar un objeto o una representación mediante una satisfacción o un descontento, sin interés alguno. El objeto de semejante satisfacción llámase bello" $(K U, \S 5)$.

Kant se distancia del empirismo porque sustituye el sujeto empírico por un sujeto trascendental, con lo que integra el juicio de gusto en su esquema trascendental y supera el límite psicológico propio de la teoría del gusto practicada por los empiristas. En la filosofía crítica, el esfuerzo por dotar de principios estables e intersubjetivos al sentimiento de placer forma parte de la búsqueda fundamental de un principio de unidad para lo diverso que dé razón de la teleología del mundo y sirva de puente entre la razón teórica y la razón práctica. Pero la teoría kantiana, por más que alcance la generalización del juicio de gusto a partir del placer desinteresado, se queda en un nivel hipotético que carece de valor epistémico. En la Crítica del juicio los juicios de gusto - reflexionantes - y los que establecen conexiones causales en la naturaleza - determinantes - se relacionan formalmente - comparten la estructura teleológica-, pero en la práctica del ejercicio de juzgar permanecen inconexos, por lo que no acaban de superar el nivel de concomitancia arriba aludido.

En el afán de preservar el valor objetivo o transubjetivo de la experiencia estética se ha revelado también útil la descripción fenomenológica, orientada a la percepción de lo que de hecho hay, de lo real en cuanto dado en la experiencia. Sin embargo, su reconocimiento implícito de la intencionalidad de la percepción no basta para superar el relativismo del juicio estético, pues es posible describir lo cultural-artístico desde una visión naturalista que no alcanza a explicar la captación de valor que comparece en la experiencia estética. La antropología cultural, por ejemplo, es prolija en un proceder descriptivo desjerarquizado en el que cualquier resto material es considerado por igual - todo es cultura, dirían Tylor y tantos otros a partir de él-, dando por sentado el relativismo y el evolucionismo ${ }^{2}$.

2 El relativismo y el evolucionismo suponen una renuncia a la intuición multisecular de que la cultura es algo distinto de la naturaleza. Según un modo de entender las cosas diverso de estas propuestas, la cultura se relaciona con la naturaleza en el sentido de que la cumple. Lejos de ser el mero conjunto acumulado de toda la producción humana, es susceptible de ordenación según el papel que cumpla en la configuración de lo humano, por más que dicha configuración no sea única, ni nunca definitiva. 


\section{Las cualidades secundarias en la tradición empirista}

Abordamos ahora la clasificación de las cualidades en primarias y secundarias que, ya presente en Descartes y Galileo, fue desarrollada y popularizada por el empirismo, especialmente a partir de la clasificación de John Locke recogida del libro II del Ensayo sobre el entendimiento humano (1610). La epistemología de Locke responde a un modelo representacionista en el que la sensación media entre las cosas y las ideas simples. Estas últimas son la base de todo el edificio del conocimiento de un modo radical, ya que Locke niega cualquier tipo de idea innata, incluidos los primeros principios, que él considera ideas $(E, \mathrm{I}, 3,1)^{3}$. Los sentidos, afectados materialmente por los objetos externos $(E$, II, $8,12)$, transmiten a la mente ideas que llamamos cualidades sensibles. Estas son de dos tipos: cualidades primarias o "ideas que adquirimos a través de más de un solo sentido [que] son las del espacio o extensión, de la forma, del reposo y del movimiento" ( $E$, II, $5,1)$; y cualidades secundarias o "poderes de producir en nosotros diversas sensaciones (...) como son colores, sonido, gustos, etc." ( $E$, II, 8,14$)$. Para diferenciarlas se vale del concepto de "semejanza". Las cualidades primarias son semejantes a los cuerpos que las producen, las secundarias son desemejantes a los mismos. Se podría decir que la cualidad primaria es la cualidad en el cuerpo, que el sujeto recibe en la idea simple como cualidad secundaria: "lo que en idea es dulce, azul o caliente, no es, en los cuerpos así llamados, sino cierto volumen, forma y movimiento de las partes insensibles de los cuerpos mismos" $(E$, II $, 8,15)$. No obstante, es importante destacar que en ninguno de los dos tipos hay identidad de la cualidad, ni con el cuerpo (primarias), ni con la idea (secundarias), sino sólo semejanza. El problema que plantea el representacionismo empirista, y que pronto se pondrá de manifiesto, es que establece una brecha entre las cosas y la mente que la idea de sensación como mediación no acaba de salvar. De hecho, ¿con qué compararemos las cualidades primarias o secundarias para poder predicar su semejanza o desemejanza respecto a su referente real, si ellas son el único modo de acceder a las cosas mismas?4 Ya en 1710 Berkeley señala en su Tratado sobre los Principios del Conocimiento Humano $(\mathrm{I}, 8)$ que las ideas sólo se pueden parecer a otras ideas.

John Locke, empeñado en dejar atrás el dogmatismo, explica el entendimiento humano estrenando una nueva terminología que refunda conceptos básicos de la tradición filosófica. Por ejemplo, habla de "cualidades" y no de "sensibles", sin diferenciar la cualidad de la cantidad e ignorando las categorías aristotélicas. Tampoco el uso que hace del término "idea" tiene nada que ver con el de la tradición platónica. Se trata de nociones nuevas cuyo significado es necesario discernir en unos textos que las usan de un modo no del todo homogéneo. De ahí cierto carácter equívoco en algunas de sus afirmaciones. En cualquier caso, su terminología es la que ha prevalecido en el tema que nos ocupa y la que emplean autores como McDowell y Wright, a quienes nos referiremos después.

3 También para Aristóteles lo sensible es la única fuente de conocimiento: "puesto que, a lo que parece, no existe cosa alguna separada y fuera de las magnitudes sensibles, los objetos inteligibles (...) se encuentran en las formas sensibles. De ahí que, careciendo de sensación, no sería posible ni aprender ni comprender (DA, 432a5).

4 En la formulación de Aristóteles, por el contrario, la diferencia entre potencia y acto permite no sólo afirmar la semejanza de la potencia sensible con su objeto, sino incluso su identidad (Cfr. DA, 431b20- 25 y 432a5). 
Aun suponiendo un modelo epistemológico distinto, la noción de cualidades primarias y secundarias coincide en sus rasgos generales con la división aristotélica entre sensibles propios y comunes y con las categorías de cualidad y cantidad. La sensación para Aristóteles consiste en una alteración cuyo objeto inmediato es el llamado sensible propio o el sensible común, siendo el primero el que es perceptible por un solo sentido, tal como "la visión del color, la audición del sonido y la gustación del sabor" (DA, 418 20), y el segundo el que es común a todos los sentidos "el movimiento, la inmovilidad, el número, la figura y el tamaño. (...) El movimiento, en efecto, es perceptible tanto al tacto como a la vista" (Ibid.). El sensible propio equivale, por tanto, a la cualidad secundaria y el sensible común a la cualidad primaria.

A su vez, cabe cierta correspondencia de la moderna "cualidad secundaria" con la categoría aristotélica "cualidad", así como de la "cualidad primaria" con la categoría "cantidad". Así lo explicitan autores como Descartes o Galileo, que utilizan esta terminología antes que Locke y Berkeley. Señalamos esta equivalencia porque permite advertir el problema que sobreviene cuando se traslada a las categorías aristotélicas el modelo de relación entre cualidades primarias y secundarias que establece el empirismo, y que parece convertir la cantidad en el contenido inteligible de la cualidad. Esta visión prescinde de que la magnitud o cantidad, por más que se manifieste mediada por la cualidad, es también un sensible (sensible común en la clasificación aristotélica), diverso de los aspectos estrictamente inteligibles ${ }^{5}$. Es importante esta aclaración por las implicaciones ontológicas que tiene: o bien se entiende la abstracción racional como de lo sensible en toda su dimensión (propio y común, cualidades primarias y secundarias) o se considera que sólo se abstrae la cualidad para reducirla a extensión. En el segundo caso se acaba por hacer coincidir esta última con la sustancia.

Tal resultado, de importantes consecuencias para la consideración de lo estético, arranca de una confusión entre el orden metodológico de las ciencias experimentales y el orden ontológico. La cantidad, que es objeto de la física matemática, es ciertamente accesible a través de la cualidad. O lo que es lo mismo, la cantidad se percibe cualitativamente. Esto implica la precedencia gnoseológica de la cualidad, entendida como cierta disposición o potencia en el objeto que por la inmutación se actualiza en la sensación, lo que no supone una correlativa precedencia ontológica por parte de la cualidad primaria. Y sin embargo, el énfasis en la objetividad de las cualidades primarias (asimilables en general a los aspectos mensurables de la sensación) como fundamento de la verdad del mundo material, unido al reduccionismo positivista, ha llevado a olvidar el valor intencional de las cualidades secundarias y, con ellas, el de la experiencia estética. El carácter relacional de la cualidad hace que las ciencias positivas prescindan de ella en su estudio de las cosas, alcanzando lo cuantitativo siempre que puede por medios experimentales más fiables. Pero afirmar el carácter disposicional de la cualidad secundaria - causa de su discontinuidad - no implica negarle realidad en la cosa o su reducción a entidad mental. La abstracción que la ciencia hace de la cualidad es

5 Que la magnitud se percibe también sensiblemente lo explica Tomás de Aquino al afirmar que "el sentido se altera de manera distinta por una superficie grande o por una pequeña, pues incluso de la blancura se dice grande o pequeña" (ST, I, 78,3). 
un recurso metodológico perfectamente legítimo que no se puede elevar justificadamente a una negación con alcance ontológico.

Como se ve, la diferenciación entre cualidades primarias o secundarias en el marco de la teoría empirista del conocimiento y vinculada al método de la ciencia moderna, deriva en la cultura positivista en una comprensión de lo estético-artístico como opuesto a lo científico y a cierto criterio de verdad.

Por ello, en nuestra búsqueda del valor real de la experiencia estética debemos revisar precisamente el contenido de la sensación, cuyo objeto como se ha dicho es la cualidad. La revisión de la clasificación de las cualidades primarias y secundarias a la que ha procedido la filosofía analítica insiste precisamente en este aspecto.

\section{La revisión de las cualidades secundarias por John McDowell}

Recurrimos en concreto a la conocida controversia entre John McDowell y Crispin Wright a propósito de la objetividad moral, que se apoya precisamente en el modelo que le presentan las cualidades secundarias.

Ambos autores, que forman parte de la tradición analítica, responsable de la pervivencia en el siglo XX de cierta herencia terminológica del empirismo, retornan a la división de lo sensible en cualidades primarias y secundarias.

McDowell se interesa por la percepción del valor, que considera inherente a la experiencia de la cualidad secundaria, como justificación del valor moral. Wright por su parte lo critica, no tanto por la consideración que hace de las cualidades secundarias como por su aplicación en el caso de la ética. Pero lo que nos interesa aquí no son las cuestiones relativas al valor moral, sino el análisis que hacen de lo que supuestamente es su modelo: la relevancia de las cualidades secundarias en la apreciación estética, inseparable de su valor intencional.

John McDowell participó en 1985 en un volumen escrito como homenaje al desaparecido J. L. Mackie, destacado defensor del escepticismo moral, con un texto titulado "Values and Secondary Qualities". En ese artículo, McDowell trata de mover el peso de la epistemología del valor de un modelo basado en las cualidades primarias, a otro sustentado por las cualidades secundarias. Lo hace mediante un diálogo crítico con el pensamiento de John Mackie, para quien "the primary-quality model turns the epistemology of value into mere mystification" $(1985,111)$. El resultado es una profunda revisión de la taxonomía de las cualidades recibida de Locke por la corrección de las ideas de subjetividad y semejanza.

McDowell parte de afirmar que si John Mackie se centra en las cualidades primarias es porque da por bueno el modelo de Locke, para quien las cualidades secundarias inducen a error a la conciencia pre-filosófica, al tender nosotros a atribuirles un papel que sólo es aplicable a las primarias. Pero esta concepción de la experiencia, entiende McDowell, es errónea. Por el contrario, señala que las cualidades secundarias tienen un valor de infalibilidad próximo al del sensible propio aristotélico, para quien sensible propio es "aquel objeto que no puede ser percibido por ninguna otra sensación y en torno al cual no es posible sufrir error" (DA, 418a20). De modo similar, afirma McDowell:

A secondary quality is a property the ascription of which to an object is not adequately understood except as true, if it is true, in virtue of the object's disposition to 
present a certain sort of perceptual appearance: specifically, an appearance characterizable by using a word for the property itself to say how the object perceptually appears. $(1985,111)$

Y continua poco después:

Secondary-quality experience presents itself as perceptual awareness of properties genuinely possessed by the objects that confront one. And there is no general obstacle to taking that appearance as face value. An object's being such as to look red is independent of its actually looking red to anyone on any particular occasion; so, notwithstanding the conceptual connection between being red and being experienced as red, an experience of something as red can count as a case of being presented with a property that is there anyway - there independently of the experience itself. $(1985,112)$

Este modo de entender la cualidad secundaria, continúa, es coherente con la definición de Locke de estas cualidades como las que tienen "powers to produce various sensations in us”. Pero McDowell diferencia la apariencia perceptiva y la causa física de la cualidad. La cualidad secundaria (la disposición en el objeto) corresponde a la apariencia perceptiva y no debe ser confundida con su causa física. Así, "[n]o doubt it is true that a given thing is red in virtue of some microscopic textural property of its surface; but a predication understood only in such terms - not in terms of how the object would look - would not be an ascription of the secondary quality of redness" $(1985,112)$. Si la causa física no es la cualidad secundaria, debe haber en esta última algo no reductible a dicha causa física.

Para Mackie, que básicamente sigue en esto a Locke, lo que justifica el calificativo de rojo es una cualidad primaria objetiva. Por lo que las cualidades secundarias siempre son reductibles a cualidades primarias. La consecuencia es que las cualidades secundarias no dicen nada. Pero, arguye McDowell, si esto fuera así, en primer lugar los colores aparecerían como neutrales, cuando en realidad la apreciación de la cualidad secundaria se experimenta siempre de modo valorativo y, en segundo lugar, los colores deberían entonces permitirnos reconstruir las cualidades primarias del objeto del que son apariencia, lo que es imposible, como demuestra el hecho de que en el laboratorio científico se considere a las cualidades secundarias como poco fiables para este tipo de reconstrucción. McDowell afirma que existe una conexión conceptual entre el hecho de que un objeto sea rojo y que aparezca como rojo a un observador y que no hay motivo suficiente para acusar a la experiencia de engañosa. Lo que justifica que yo llame rojo a un objeto, repetimos, no es nada distinto de su capacidad de aparecer como rojo.

En esta argumentación, como se ve, McDowell está reivindicando la intencionalidad y el valor apreciativo que acompaña siempre la percepción de la cualidad secundaria. De modo que hay una especificidad en la cualidad secundaria que está vinculada, no con la propiedad física del objeto, sino con el valor, que corresponde a otro tipo de conocimiento o experiencia. La percepción del valor que acompaña la experiencia de las cualidades secundarias es, según él, su modo específico de ser, aquello no reductible a las cualidades primarias.

Para justificar la objetividad del juicio de valor estético (que pretende, como veremos, aplicar al juicio ético), McDowell tiene que superar la idea de que las cualidades secunda- 
rias inducen a error. Idea que se ha recibido del empirismo como un contenido inseparable de su propia definición porque está implícito en la idea de desemejanza. Ya hemos visto cómo insiste en afirmar algo así como la infalibilidad de los sensibles propios. Se detiene también a explicar en qué sentido la percepción de las cualidades secundarias es subjetiva. Comenta McDowell que entender lo subjetivo como irreal o ilusorio es un error típico de Locke, asumido por autores como Mackie.

Now this contrast between objective and subjective is not a contrast between veridical and illusory experience. But it is easily confused with a different contrast, in which to call a putative object of awareness 'objective' is to say that it is there to be experienced, as opposed to being a mere figment of the subjective state that purports to be an experience of $i^{6}$. $(1985,113-114)$

¿Por qué lo subjetivo puede ser real?, porque la relación entre cómo son las cosas y cómo aparecen, a diferencia de lo que afirma el representacionismo empirista, no es una relación de semejanza, sino - cuando es verídica - de identidad (1985, 115). Encontramos de nuevo aquí la afirmación de algo ya presente en Aristóteles, para quien el cognoscente y lo conocido son uno en acto. Como se ha dicho arriba, la cualidad secundaria se da en el cognoscente en virtud de la operación. En McDowell, la experiencia subjetiva no es opaca, lo aparente o fenoménico no es su límite, sino que está abierta a la realidad.

If we are to achieve a satisfactory understanding of experience's openness to objective reality, we must put a more radical construction on experience's essential subjectivity. And this removes an insidious obstacle (...) that stands in the way of understanding how secondary- quality experience can be awareness, with nothing misleading about its phenomenal character, of properties genuinely possessed by elements in a not exclusively phenomenal reality. $(1985,117)$

Una vez señaladas en las cualidades secundarias las notas de infalibilidad, intencionalidad, valor e irreductibilidad, McDowell da el salto de la estética a su principal punto de interés, que es la ética. La argumentación que desarrolla en este sentido nos interesa aquí por la crítica que contiene al proyectivismo como único método de verificación, que afecta también a las afirmaciones de Crispin Wright a las que nos referiremos después. En el método proyectivista, indica McDowell, "having one's ethical or aesthetic responses rationally suited to their objects would be a matter of having the relevant mechanism functioning acceptably" (1985, 122). Es decir, la verificabilidad se basa en una descripción adecuada del proceso que exige al sujeto situarse en un imposible punto de partida externo al juicio. Pero la percepción de la cualidad secundaria y la valoración que cuaja en el juicio estético no responde a ningún proceso. McDowell critica que, en cuestión de ética o estética, este tipo de objetivación termina cayendo en definiciones externalistas de lo bello y de lo bueno.

6 En un trabajo anterior McDowell había abordado con más detenimiento la crítica a la noción de objetividad que emplea Mackie en su argumentación, poniéndola en relación con las tesis de Bernard Williams (Cfr. McDowell, 1983). 
How, then, are we to understand this pictured availability of the processing mechanism as an object for contemplation, separated off from the world of value? Is there any alternative to thinking of it as capable of being captured, at least in theory, by a set of principles for superimposing values on to a value-free reality? The upshot is that the search for an evaluative outlook that one can endorse as rational becomes, virtually irresistibly, a search for such a set of principles: a search for a theory of beauty or goodness. One comes to count 'intuitions' respectable only in so far as they can be validated by an approximation to that ideal. $(1985,122)$

Este es el resultado del intento de objetivar la subjetividad. A este método proyectivista McDowell opone un modo internalista que sería el adecuado para los juicios de tipo ético o estético. Según él los valores, del mismo modo que las cualidades secundarias, no son inteligibles fuera de la experiencia que se tiene de ellos, por lo que la atribución de valor ético a una acción surge, como en el caso de la percepción de la cualidad, de cierta modificación del sentido o la sensibilidad $(1985,117)$. La imposibilidad de error en el caso de la cualidad secundaria es transferida luego al juicio ético para alcanzar una forma de verificación no cognitivista con la que responder al escepticismo empirista de Mackie.

La alternativa al proyectivismo que McDowell propone le sirve tanto para evitar el anything goes como la generalización de los valores. Su posición cuestiona la posibilidad de establecer una teoría normativa del buen gusto ético y estético, pero está igualmente lejos de defender la arbitrariedad inapelable del gusto subjetivo.

\section{El modelo de verificación proyectivista de Crispin Wright}

La idea de una verificación del juicio no cognitivista de McDowell fue contestada en 1992 por Crispin Wright, quien en Truth and Objectivity intentó llevar las tesis de McDowell a un modelo proyectivista, tratando de estandarizar, por un lado, al sujeto que emite el juicio $\mathrm{y}$, por otro, la experiencia de la que emerge. Wright reconoce con McDowell que es necesario superar a Locke y dejar de identificar la subjetividad de las cualidades secundarias con su carácter ilusorio o falto de realidad7. Acepta también la imposibilidad de una verificación "a posteriori", pero intenta llegar a una determinación de las condiciones "a priori”. Conviene detenerse brevemente en este intento para mostrar cómo termina de nuevo en otra forma de normalización o estandarización del juicio estético que deshace en cierto modo el carácter siempre particular y "creativo" que McDowell había acertado a ver.

El capítulo de Truth and Objectivity titulado "The Euthyphro Contrast" se ocupa expresamente de la teoría de las cualidades secundarias, dejando de lado su valor ontológico para centrarse en los aspectos lógicos o predicamentales de la cualidad. Wright valora los intentos de autores como McDowell por superar el escepticismo antirrealista, pero insiste en la necesidad de formalizar un método de verificación para las cualidades secundarias aceptable en el marco de la filosofía analítica.

7 En este sentido admite Wright: “an object's secondary qualities constitute material for cognition” (2003, 156157). 
En el diálogo platónico Eutifrón, Sócrates presenta a Eutifrón el dilema entre dos alternativas que Wright convierte en modelos de verificación. A saber:

- Algo es piadoso porque Dios lo aprueba, o bien,

- lo que es piadoso, Dios lo aprueba.

La primera, correspondiente a la opción del sofista Eutifrón, seguiría un modelo que Wright llama "proyectivista", aplicable a las cualidades secundarias; la segunda, defendida por Sócrates en el diálogo platónico, equivaldría a un modelo de verificación "detectivista", adecuado para las cualidades primarias. Es decir el peso probatorio del juicio del sujeto sobre lo sensible es diverso en uno y otro caso. El tipo de proposición que expresa el juicio sobre una cualidad secundaria (o cualidad, a secas) según Wright es un bicondicional del tipo " $x$ is $Q$ iff for any $S$ : if $S$ were perceptually normal and were to encounter $x$ in perceptually normal conditions, $S$ would experience $x$ as $Q$ ”.

Si $Q$ es una cualidad secundaria, la mejor opinión (es decir, el juicio realizado en condiciones de percepción estándar por un sujeto con capacidad perceptiva estándar) es la base conceptual para atribuir la verdad dentro de un discurso. En este caso la atribución de verdad es necesaria. En el caso de que $Q$ sea primaria (modelo detectivista), la mejor opinión no determina necesariamente la verdad, por lo que la atribución de verdad a lo propuesto por $S$ es contingente.

Por consiguiente, en este planteamiento es fundamental establecer las condiciones que permiten entender si el juicio particular sobre una cualidad secundaria representa la mejor opinión, y para ello se procede a la determinación suficiente de las condiciones que ha llamado "estándar", que según él deben ser describibles. El resultado es, finalmente, que no se encuentra un criterio mejor para establecer esas condiciones que la mera estadística, de modo que "it is the judgements of those who are actually statistically standard observers, in what are actually statistically standard conditions of observation, which count" (1992, 114). La falta de una descripción más segura de la "mejor opinión", obliga a conceder que la diferencia entre cualidades primarias y secundarias no puede radicar en la contingencia o la necesidad del juicio del sujeto, tal como había establecido al comienzo de su argumentación, puesto que no es posible atribuir necesidad a un modelo estadístico. La diferencia entonces consiste en que las condiciones estándar para el mejor juicio sobre una cualidad, en el caso de las secundarias, pueden ser establecidas a priori.

Así, estableciendo que las cualidades secundarias, dependientes de respuesta (responsedependent), son verificables según su concordancia con verdades construidas a priori (1992, 136), y al hacer de dichas construcciones a priori un modelo externo de verificación, Wright cae de nuevo en el tipo de objetivación heterónoma que, según McDowell, no es aplicable a las valoraciones estéticas o morales.

Podemos entonces concluir que la formalización de un modelo analítico de verificación para la predicación de las cualidades secundarias no ayuda a ampliar el estrecho campo de certezas que permite alcanzar el positivismo. Y es que, como se ha señalado, el tipo de verdad atribuible al juicio estético no puede depender de proposiciones unívocas y bivalentes, entre otras cosas porque mantiene siempre su dependencia del carácter particular y posicionado de la experiencia. Se trata entonces de un tipo de verdad cuya predicación no 
es verificable de modo teórico, similar en esto a la verdad práctica que se dice de la acción moral. Otra cosa es que sea pertinente el paralelismo que establece McDowell entre el tipo de reacción sensible que comparece en uno y otro tipo de juicio (el moral y el estético). Sin entrar a fondo en esta disquisición, baste señalar que para Wright no existe, en el juez que decide sobre lo bueno, un sistema receptor seguro e infalible (que sí reconoce por tanto en el caso de las cualidades secundarias), sino una competencia adquirida ${ }^{8}$. En línea con la crítica de Wright cabe añadir que la "naturalidad" con la que la sensibilidad reacciona al valor ético no exige ser explicada mediante la existencia de un problemático "sentido moral", puesto que la noción de "segunda naturaleza" propia del concepto aristotélico de virtud ya da cuenta de ello de modo suficientemente satisfactorio.

\section{Cualidad secundaria y verdad}

Estamos ya en condiciones de dar un paso más en nuestra búsqueda del valor epistémico de la cualidad secundaria. Si, como hemos visto, la cualidad secundaria cuenta con un sistema receptor seguro e infalible, intencional, que no es sólo material para el conocimiento racional abstracto, podemos ya preguntarnos qué otro tipo de noticia nos trae. Dicho de otro modo, ¿de qué se predica esa verdad encontrada en la experiencia estética?, puesto que no se dice de la acción, contenido del tipo de verdad (práctica) a la que, al descartar su verificabilidad lógica, hemos dicho que se parece la del juicio estético.

En primer lugar, partimos de reconocer que existe en la cualidad secundaria un algo previo distinguible de los conceptos. En Wright se trata de un material que se registra en la conciencia, pero que no tiene valor epistémico alguno mientras no comparece el concepto. La especificidad que Wright encuentra en la sensación de las cualidades secundarias se limita al registro "crudo" (raw) que no lleva noticia alguna si no es "cocinado" con el concepto9. Por su parte, McDowell ha continuado en toda ocasión defendiendo la epistemología de las cualidades secundarias. Así, por ejemplo, en su Aquinas Lecture de 2011, publicada con el título Perception as a Capacity for Knowledge.

En segundo lugar, consideramos la defensa que McDowell hace de la valoración como un aspecto intrínseco y definitorio de la percepción cualitativa. Para explicarla nos serviremos

8 En 2003 Wright publicó Saving the Differencies, en cuyo capítulo "Moral Values, Projection and Secondary Qualities" (pp. 155-182) criticaba la comparación entre cualidades secundarias y valores que McDowell había puesto de moda en los ochenta, porque implica la existencia de un tipo especial de experiencia que sería la experiencia moral, con una especificidad análoga a la que existe para experimentar las cualidades secundarias. La idea de la existencia de un sentido moral ha sido común desde Hume, que decía: "When you pronounce any action or character to be vicious, you mean nothing, but that from the constitution of your nature you have a feeling or sentiment of blame from the contemplation of it. Vice and virtue, therefore, may be compared to sounds, colours, heat and cold, which according to modern philosophy, are not qualities in objects but perceptions in the mind" $(2003,156)$.

9 Lo explica mediante el ejemplo de la reacción de un bebé a un ruido intenso. Y concluye: "If our experience of secondary qualities provides a model of anything, then it is of a notion of experience which is, up to a point at least, raw. (...) It is not merely what we are able to say or judge about it but the experience itself what actually registers in consciousness, which depends, in some measure, on the concepts brings to bear. The point remains: if our experience of secondary qualities is to be the model, then not everything in the manifest character of an experience can be seen as owed to concepts brought to bear" $(2003,167)$. 
de la teoría aristotélica de los sentidos, la idea de proporción y su vinculación con la órexis o la estimativa. A continuación explicamos estas nociones, que clarifican y soportan aspectos principales de lo que McDowell defiende.

Como es sabido, Aristóteles define el sentido como una cierta proporción, pero entender correctamente esta definición exige enmarcarla en algunas distinciones previas. En primer lugar, hay que diferenciar lo percibido sensiblemente - propiamente la cualidadde la cosa: "el sentido sufre también el influjo de cualquier realidad individual que tenga color, sabor o sonido, pero no en tanto que se trata de una realidad individual, sino en tanto que es de tal cualidad y en cuanto a su forma". En segundo lugar, dentro del sentido diferencia la potencia del órgano: "El órgano primario es, por su parte, aquel en el que reside semejante potencia". Aclara no obstante que la diferencia entre ambos (potencia sensible y órgano) es inteligible, no real:

Desde luego que la potencia no se distingue realmente del órgano, pero su esencia es distinta: en caso contrario, el ser dotado de sensibilidad sería, en cuanto tal, una magnitud; y, sin embargo, ni la esencia de la facultad sensitiva ni el sentido son magnitud, sino más bien su proporción idónea y su potencia $(D A, 424 \mathrm{a} 25)$.

Este último párrafo indica, en primer lugar, que lo que define como proporción es la esencia de la facultad o potencia sensitiva, y en segundo lugar que la proporción no es magnitud (lo que llevaría de nuevo a la reducción de la cualidad cuestionada más arriba), sino el sentido de su adecuación. Ahora bien, es sabido que la causa de este sentido de adecuación - o proporción - se suele llamar armonía. Definir el sentido como proporción, y ésta como adecuación introduce la apreciación en la esencia del mismo. Porque, ¿qué es el sentido de la adecuación sino ese afecto o apreciación valorativa que se manifiesta en el placer ${ }^{10}$ (o en su contrario)? Podemos decir ya entonces que la percepción sensible manifiesta - actualiza - nuestra adecuación con el mundo ${ }^{11}$, lo que es coherente con el carácter relacional de la cualidad y con su intrínseco sentido valorativo. La sensación de la cualidad por tanto, aun dependiente de la relación, tiene carácter epistémico y da noticia precisamente de esa relación, pues informa tanto sobre el sujeto como sobre el mundo.

A diferencia de lo que algunos entendieron, la idea aristotélica de armonía, que se manifiesta en la sensación/proporción como placer o displacer, no permite para lo estético el tipo de objetivación normativa que McDowell reprobaría por heterónoma. La proporción es susceptible de modificación pues, como se ha dicho, la alteración de la sensación es origen de procesos transitivos - y no sólo de operaciones inmanentes - : la capacidad de

10 De hecho, en Aristóteles es el placer lo que vincula la sensación con el deseo (órexis), lo que no implica contradicción con la idea moderna de placer desinteresado. Tomás de Aquino nombra de modo diverso la órexis en los seres irracionales y en los racionales: la estimativa como relativa al instinto animal, la cogitativa relacionada con la capacidad humana de establecer comparación entre lo almacenado en la memoria. Dicha distinción, igualmente aplicable a la consideración de un placer desinteresado de la existencia de su objeto, sirve también a la defensa del libre arbitrio $(S T, \mathrm{I}, 78,4)$.

11 En esta afirmación está implícito el reconocimiento de la apertura universal de la sensibilidad, que es a su vez necesario en la defensa de la sensibilidad como fuente única de conocimiento. En Tomás de Aquino, la clasificación de los sentidos, en razón de la semejanza que permite la relación cognoscitiva, responde a una clasificación previa y real de las cualidades existentes (Cfr. ST, I, 87,1). 
placer o disgusto se va ampliando gracias a las mediaciones culturales que modifican constantemente nuestro modo de relación con el mundo. La adecuación se capta como valor por la inmediación de la sensación o por la expansión de las posibilidades de adecuación que permite la mediación cultural. Por eso, a pesar de que el límite orgánico - el exceso o defecto de lo que podemos ver o no ver, oír o no oír- es objetivable, la proporción del sentido que define la armonía no lo es, o al menos no es universalizable.

De modo que aquel "afecto pre-comprensivo" de que se habló al principio, que McDowell relaciona con la captación de valor y que sería lo específico de la experiencia estética, se explica y clarifica a partir de la definición del sentido como proporción. Esta apreciación coincide con lo particular de la sensación, con un material que no se podría llamar crudo, si por crudo entendemos neutro. Si cabe pensar la experiencia estética desde la felicidad o el placer, como hace la teoría estética moderna, es por la unidad del síntoma - el placer-con lo que significa, cierto sentido de adecuación o conveniencia con el mundo que es tan real como relacional.

\section{Conclusión}

En las páginas precedentes nos hemos interesado por el estudio de las cualidades secundarias como un medio para desatar la circularidad que nace de la mutua dependencia entre estética y teoría del arte.

Nos hemos servido para ello del enfoque de John McDowell que, por un lado, reivindica al igual que Wright la intencionalidad que Locke y Mackie niegan a las cualidades secundarias, y que, por otro, señala la apreciación como elemento intrínseco y no añadido a la percepción de la cualidad.

La comparación con la teoría aristotélica de los sensibles propios y del sentido entendido como proporción refuerza la intuición contenida en las tesis de McDowell, en cuyo enfoque la percepción sensible queda definida por sus notas de intencionalidad y valoración.

La disolución de la problemática interdependencia de arte y estética a partir de este enfoque se sustenta en dos premisas, asumidas a lo largo de la exposición precedente. En primer lugar, que la estética es el estudio de la aísthesis, aspecto de la experiencia cuyo principio es la percepción de la cualidad. En segundo lugar, que lo estético es el principal elemento definitorio de lo que llamamos "artístico". Hecho esto, a partir del análisis realizado de las cualidades secundarias es posible aventurar la siguiente hipótesis, que deberá ser abordada en estudios subsiguientes: que la "noticia" sensible sobre la adecuación o inadecuación de sujeto y mundo sea la base, esto es, el mínimo necesario, sobre el que establecer el valor artístico.

El arte y la estética han sido relegados en su valor epistémico por la fría razón productiva heredera de la modernidad. Este haber sido relegados encuentra en nuestra "vida alienada" su expresión positiva en el anhelo de mediaciones que nos permitan recuperar el sentimiento de la adecuación. En la necesidad de poseer el presente, sin dilapidarlo en anticipaciones y aplazamientos, concurre la experiencia estética, que no viene para cubrir con un velo de cualidad y placer la dura realidad cuantitativa, sino a desvelar la verdad de nuestro lugar en el mundo, la posibilidad de habitarlo adecuadamente. 


\section{Referencias bibliográficas}

Aristóteles, (2000) Acerca del alma (citado como DA), Traducción de Tomás Calvo Martínez, Gredos.

Kant, I. (2007), Crítica del juicio (citado como $K U$ ), traducción de Manuel García Morente, Tecnos.

Locke, J. (1956), Ensayo sobre el entendimiento humano (citado como E), traducción directa de Edmundo O'Gorman, Fondo de Cultura Económica.

McDowell, J. (1983), "Aesthetic Value, Objectivity, and the Fabric of the World", en Eva Schaper (ed.), Pleasure, Preference and Value. Studies in Philosophical Aesthetics, Cambridge U. Press, pp. 1-16.

McDowell, J. (1985), "Values and Secondary Qualities", en Ted Honderich (ed.), Morality and Objectivity. A Tribute to J.L. Mackie, Routledge \& Kegan Paul, pp. 110-129.

McDowell, J. (2003), Mente y mundo, Ediciones Sígueme.

McDowell, J. (2011), Perception as a Capacity for Knowledge, Marquette University Press.

Tomás de Aquino (1956), Suma Teológica (citado como ST), BAC.

Wright, C. (1992), Truth and Objectivity, Harvard University Press.

Wright, C. (2003), Saving the differences. Essays on themes from "Truth and Objectivity", Harvard University Press. 